\title{
Coherent control of boosted terahertz radiation from air plasma pumped by femtosecond three-color sawtooth field
}

Shaojie Liu, ${ }^{1}$ Zhengquan Fan, ${ }^{1}$ Chenhui Lu, ${ }^{2, ~}{ }^{\#}$ Jieyu Gui, ${ }^{1}$ Cheng Luo, ${ }^{1}$ Shixiang Wang, ${ }^{1}$ Qingqing Liang, ${ }^{1}$ Bin Zhou, ${ }^{1}$ Aurelién Houard, ${ }^{3}$ André Mysyrowicz, ${ }^{3}$ Vasily Kostin, ${ }^{4,5}$ Yi Liu ${ }^{1,6, *}$

${ }^{1}$ Shanghai Key Lab of Modern Optical System, University of Shanghai for Science and Technology, Shanghai, 200093, China

${ }^{2}$ School of Mechanical and Automotive Engineering, Shanghai University of Engineering Science, Shanghai, 201620, China

${ }^{3}$ Laboratoire d'Optique Appliquée, ENSTA Paris, CNRS, Ecole Polytechnique, Institu Polytechnique de Paris, 828 Boulevard des Maréchaux, 91762 Palaiseau Cedex, France

${ }^{4}$ Institute of Applied Physics, Russian Academy of Sciences, Nizhny Novgorod 603950, Russia

${ }^{5}$ University of Nizhny Novgorod, Nizhny Novgorod 603950, Russia

${ }^{6}$ CAS Center for Excellence in Ultra-Intense Laser Science, Shanghai 201800, China

\#1chhuiz@163.com,*yi.liu@usst.edu.cn

\begin{abstract}
:
We report on the coherent control of boosted ultrashort terahertz pulse emitted from air plasma pumped by a femtosecond sawtooth pulse composed of three optical harmonic fields. With an in-line optical setup, the relative phases between the three optical components of the pump pulse can be varied independently with attosecond precision without the complexity of external phase stabilization. We observe that the amplitude of the terahertz emission pumped by the phase-optimized sawtooth wave is enhanced by 1.8 times compared to the widely used two-color scheme. Moreover, by manipulating the relative phases between the three colors at attosecond precision, coherent control of the azimuthal angle, ellipticity, and polarity of $\mathrm{THz}$ radiation is achieved. A local current model reproduces well the coherent control observations.
\end{abstract}




\section{Introduction}

Air plasma created by ultrafast laser pulses is a unique source of coherent terahertz radiation because of its extremely broad bandwidth [1-4]. It reaches up to $200 \mathrm{THz}$, while other commonly used broadband $\mathrm{THz}$ radiation sources such as optical-conductive antenna or optical rectification crystal are usually limited to be below $5 \mathrm{THz}$ [5-7]. It is well known that the combination of a fundamental femtosecond laser pulse with its second harmonic (2-color scheme) can produce $\mathrm{THz}$ radiation with a conversion efficiency of $10^{-4}$, two orders of magnitude higher than the single-color excitation [3, 5, 8-10]. With this two-color scheme, $\mathrm{THz}$ fields with amplitude exceeding $1 \mathrm{MV} / \mathrm{cm}$ have been demonstrated [11, 12]. Moreover, the polarization and spectrum of the THz pulse can be controlled by varying the polarization, duration, and spectrum of the two-color pulses [13-19]. The THz generation mechanism has been first interpreted as a four-wave mixing process [2, 13], and later attributed to a transient residual electron currents inside the plasma [3, 9, 16, 19-22]. With 2-color optical driven field, a net transient transverse electron current is effectively produced, since the symmetric electron motion in case of single-color pumping is broken [3, 20-24]. Predicted in [23] and experimentally demonstrated in [24], $\mathrm{THz}$ generation by two-color field with uncommon commensurate frequencies has confirmed that transient electron current is the dominating mechanism for such $\mathrm{THz}$ emission.

To further increase the terahertz yield, it was theoretically proposed to use three- and multicolor pumping fields $[18,25]$. Particularly, it was predicted that a sawtooth wave would be beneficial since such an optical field can increase the transient electron current by maximizing the electron drift velocity [25]. Numerical simulations show that THz energy can be significantly enhanced by such an engineered phase-optimized optical field [25-27]. The sawtooth-like wave can be synthesized by three or more optical fields with frequencies forming a series of harmonics, with their relative phase alternatively varied by $\pi$ [25]. Unfortunately, active control and stabilization of the relative phases between $N$-color optical fields $(N \geq 3)$ in experiments are not all trivial and normally require complex feedback loops with attosecond control precision based on interferometer phase measurement [28, 29]. Therefore, THz generation using a femtosecond sawtooth-like wave has not yet been experimentally demonstrated, although $\mathrm{THz}$ enhancement has been observed with three-color fields obtained from Optical Parametric Amplifier (OPA), where the relative phases between the optical fields are usually not fixed [30, 31].

In this work, we demonstrate a simple inline optical setup which enables attosecond control of the relative phases between three optical harmonic fields, without the need of any external phase stabilization system. Our method is based on the femtosecond laser pulses at $800 \mathrm{~nm}$ wavelength, 
which is widely available for the laboratories engaged in ultrafast optics. With optimized relative phases, we find that the $\mathrm{THz}$ pulse amplitude obtained with the three-color sawtooth-like wave is 1.8 times higher than that of two-colors pumping. By adjusting the phases between the three colors, we show that this boosted terahertz emission can be coherently controlled in terms of azimuthal angle, polarity and amplitude. We simulate this coherent control process with a local current model that reproduces well the observations. In particular, we show that the phase period for $\mathrm{THz}$ intensity variation now becomes $2 \pi$ due to the absence of mirror-symmetry of the sawtooth wave.

\section{Setup for the sawtooth-like wave generation and terahertz field detection}

For synthesis of the three-color femtosecond sawtooth-like wave, we used the optical setup schematically presented in Fig. 1. Femtosecond laser pulses first pass through a $200 \mu \mathrm{m}$ thick type I Beta Barium Borate (BBO) crystal cut at $29.2^{\circ}$ for second harmonics generation, which is followed by a calcite phase compensator, a dual-wavelength half-wave plate, and a pair of fused silica optical wedges. This optical layout is commonly used for two-color field coherent synthesis [32]. By displacing the fused silica wedges, the change of fused silica thickness $\Delta d$ induces a phase variation $\Delta \varphi=2 \pi\left(n_{800}-n_{400}\right) \Delta d / \lambda_{400}$, where $n_{800}$ and $n_{400}$ are the refractive indices of fused silica for $800 \mathrm{~nm}$ and $400 \mathrm{~nm}$ wavelength respectively. It should be noticed that a change of transverse position of the moving wedges by $\Delta x=10 \mu \mathrm{m}$ results in a change of the wedges thickness of $\Delta d=\Delta x \tan \theta=0.524 \mu \mathrm{m}$, corresponding to $\Delta \varphi=0.044 \pi$. Here $\theta=3^{\circ}$ is the angle of the fused silica wedge. This leads to a time delay of 29.3 attoseconds between the $800 \mathrm{~nm}$ and $400 \mathrm{~nm}$ fields. Therefore, $\Delta \varphi$ can be easily controlled with attosecond precision in this simple setup. The synchronized $800 \mathrm{~nm}$ and $400 \mathrm{~nm}$ fields, linearly polarized in the horizontal plane, are focused in air by a parabolic mirror. Before the focus, a $50 \mu \mathrm{m}$ thick type I BBO crystal for Sum Frequency Generation (SFG) is inserted in the beam path in order to produce the third harmonics at $266 \mathrm{~nm}$.

After the SFG crystal (BBO 2), the 800, 400 and $266 \mathrm{~nm}$ normally have both horizontal and vertical components due to the birefringence of the crystal. The relative phase between the 400 $\mathrm{nm}$ and $266 \mathrm{~nm}$ at the focus contains a constant phase component determined by the thickness of the BBO 2 and a variable phase $\gamma=2 \pi\left(n_{400}-n_{266}\right) l / \lambda_{266}$, where $l$ is the BBO-2-focus distance, $n_{400}$ and $n_{266}$ are the refractive indices of air for 400-nm and 266-nm pulses. Therefore, one can change the position of the BBO 2 to control $\gamma$. A change of $l$ by $10 \mu \mathrm{m}$ corresponds to a phase change of $\Delta \gamma=0.0011 \pi$. In the Appendix, we presnet a detailed consideration of the physical sources contributing to the phase $\varphi$ and $\gamma$. In the inset of Fig. 1, we present a comparison of a 3-colors sawtooth-like wave and the 2-colors wave to illustrate their difference. 
Under the focusing role of the $f=150 \mathrm{~mm}$ parabolic mirror, the 3-color wave was focused to form a plasma in air or nitrogen gas. Femtosecond pulses with $40 \mathrm{fs}$ duration and pulse energy of $1.8 \mathrm{~mJ}$ before the first $\mathrm{BBO}$ crystal was used in the experiment. The emitted $\mathrm{THz}$ emission from the gas plasma was measured with the Electronic-Optical Sampling (EOS) technique in a $1 \mathrm{~mm}$ thick ZnTe crystal. By rotation of the detection crystal and the probe polarization, we can measure both horizontal and vertical components of the THz field. The experimental system was immersed in pure nitrogen to avoid the water vapor absorption of $\mathrm{THz}$ radiation.

\section{Experimental results}

We first compared the $\mathrm{THz}$ emissions obtained by two-color and three-color sawtooth wave excitation. For the two-color case, the insertion of the fused silica wedge was optimized for the most intense THz generation [3]. The energy of the incident $800 \mathrm{~nm}$ pump pulse was $1.6 \mathrm{~mJ}$ and the second harmonic was $0.2 \mathrm{~mJ}$. The measured THz waveform is presented in Fig. 2(a) (red line). Since both $800 \mathrm{~nm}$ and $400 \mathrm{~nm}$ optical fields are linearly polarized in the horizontal plane (Fig. 3(a)), the resulting THz electric field is observed to be horizontally polarized with a weak vertical component, in agreement with previous results [13]. For the three-color field excitation, it is observed that both the rotation and azimuth angle of the SFG crystal determine the intensity of the $266 \mathrm{~nm}$ field, as well as the polarization state of the exiting three optical fields. Moreover, the distance of the SFG BBO crystal with respect to the focus determines the phase $\Delta \gamma$. In general case, the resulting THz emission exhibits both $x$ and $y$ components, with their amplitude and phase determined by the three-color optical fields. In the experiments, we optimize both the rotation and azimuth angle of the SFG crystal, as well as the relative phases $\Delta \varphi$ and $\Delta \gamma$, to achieve the most energetic THz emission. In Fig. 2(b), we present one such optimized THz field. The THz electric field now presents elliptical polarization with both horizontal and vertical components. The total amplitude of this optimized THz field is found to be 1.8 times higher than that of the 2-color pumping, corresponding to an energy enhancement of 3.2 times. The corresponding polarization of the 800, 400, and $266 \mathrm{~nm}$ optical fields are presented in Fig. 3 (b).

We now examine the role of the relative phases $\Delta \varphi$ and $\Delta \gamma$. We present in Fig. 2(c) the profile of the THz electric field oscillation for a systematic variation of the phase $\Delta \varphi$. The azimuthal angle of the THz radiation, as well as its ellipticity, are observed to be controlled by this phase factor. The phase $\Delta \gamma$ was also changed by displacing the SFG crystal and the resulting THz field profile are shown in Fig. 2(d). In this case, the THz field is found to be gently modified by the change of $\Delta \gamma$ in terms of the azimuthal angle and the ellipticity.

To get insight into the underlying physics mechanism and simplify the measurements, below we concentrate ourselves to one dimension of the $\mathrm{THz}$ field, namely, the vertically (y) polarized 
terahertz component. In Fig. 4, we present a systematic measurement of temporal waveform of the vertically polarized THz field in the time domain by varying $\Delta \varphi$, where five different $\Delta \gamma$ were chosen. As seen, we coherently control the amplitude and polarity of the $\mathrm{THz}$ pulse by manipulation of $\Delta \varphi$. As an example, in Fig. 5(a) we present two representative THz wave forms with opposite polarities, achieved by $\Delta \varphi=0$ and $\Delta \gamma=0.78 \pi$ versus $\Delta \varphi^{\prime}=\pi$ and $\Delta \gamma^{\prime}=0.22 \pi$. We present the squared modulus of THz peak-to-peak amplitude $\left|U_{T H z}\right|^{2}$, which is proportional to the THz pulse energy, as a function of $\Delta \varphi$ in Fig. 5(b). Here, a $2 \pi$-periodic variation of the THz energy is observed for all five different $\Delta \gamma$. The experimental results in Fig. 5(b) are well reproduced by numerical simulation, as presented in Fig. 5(c), which will be discussed later.

We next studied the role of $\Delta \gamma$, while the phase $\Delta \varphi$ was fixed at three chosen values. Systematic experimental results of the $\mathrm{THz}$ waveform are presented in Fig. 6. It is interesting to notice that the variation of BBO-2-focus distance $(\Delta \gamma)$ does not necessary lead to polarity change of the THz pulse, although its amplitude can be strongly modulated. We present in Fig. 7(a) the THz amplitude as a function of the BBO-2-focus distance. A periodic oscillation with a period of the BBO-2-to-focus distance of $18.0 \mathrm{~mm}$ was observed for all three chosen $\Delta \varphi$, corresponding to a $2 \pi$ change of $\Delta \gamma$. The maximum $\mathrm{THz}$ amplitudes correspond to values of $\Delta \varphi$ differing by $\mathrm{n} \pi(\mathrm{n}=0, \pm 1, \pm 2, \ldots)$, while the polarity of THz pulse remains unchanged for varying $\Delta \gamma$. For other values of $\Delta \varphi$, THz polarity change can also be observed, for example in the case where $\tau$ $=0.352 \mathrm{fs}$ (corresponding to $\Delta \varphi=0.53 \pi$ ) as shown in Fig. 6(b) and Fig. 7(a).

\section{Discussion and numerical simulations}

To corroborate our experimental findings, we utilized the local current (LC) model while neglecting the propagation effects $[3,19,20,24,25]$. The electric field projection $E(t)$ in linearly polarized three-color laser pulse is expressed as

$E(t)=\exp \left(\frac{-2 \ln 2 t^{2}}{\tau^{2}}\right) \times\left[E_{800} \sin \left(\omega_{0} t\right)+E_{400} \sin \left(2 \omega_{0} t+\pi+\Delta \varphi\right)+E_{266} \sin \left(3 \omega_{0} t+\Delta \varphi+\Delta \gamma\right)\right]$

where $t$ is the time variable, $\tau$ is the intensity full width at half-maximum, $\omega_{0}=2 \pi c / \lambda_{800}$ is the fundamental frequency. $E_{800}, E_{400}$ and $E_{266}$ are the field amplitudes of the fundamental, second and third harmonic laser field, respectively. More details concerning Eq. (1) can be found in the Appendix. For standard sawtooth-like pulse, the amplitude ratios should be set to $E_{400}=E_{800} / 2$ and $E_{266}=E_{800} / 3$ and the phase shifts $\Delta \varphi$ and $\Delta \gamma$ should be set to zeros. In our experiment, the pulse energies of the $400 \mathrm{~nm}$ and $266 \mathrm{~nm}$ are measured to be $230 \mu \mathrm{J}$ and $22.5 \mu \mathrm{J}$, respectively. However, due to the intensity clamping effect inside the plasma for the fundamental $800 \mathrm{~nm}$ pulse and the strong defocusing effect of the plasma on the $400 \mathrm{~nm}$ and $266 \mathrm{~nm}$ pulses, the precise laser intensities of the three optical pulses cannot be identified easily. Therefore, in our 
simulations, we have tested a vast range of laser intensity ratio between the three optical fields and we found the best agreement between experiments and simulations is obtained in case of $E_{400}=E_{800} / 4$ and $E_{266}=E_{800} / 5$.

For the fundamental laser intensities in our experimental conditions $\left(10^{14}-10^{15} \mathrm{~W} / \mathrm{cm}^{2}\right)$, the tunneling formula can be employed to calculate the ionization rate $W(t)$ [33]:

$$
W(t)=\frac{\alpha}{|\varepsilon(t)|} \exp \left[-\left(\frac{\beta}{|\varepsilon(t)|}\right)\right]
$$

where $\varepsilon(t)=E(t) / \varepsilon_{a}$ is the electric field in atomic units with $\varepsilon_{a}=5.14 \times 10^{9} \mathrm{~V} / \mathrm{cm}, \alpha=$ $4 \omega_{a} r^{5 / 2}, \beta=(2 / 3) r^{3 / 2}, \omega_{a}=4.13 \times 10^{16} \mathrm{~s}^{-1}$ is the atomic frequency, and $r$ is the ionization potential of the gas molecule relative to hydrogen atom $r=U / U^{H}$. In our simulations, we used $U=15.6 \mathrm{eV}$ (for $\mathrm{N}_{2}$ gas) and $U^{H}=13.6 \mathrm{eV}$. The free electron density $N_{e}(t)$ can be written as

$$
\frac{d N_{e}(t)}{d t}=W(t)\left[N-N_{e}(t)\right],
$$

where $N$ is the initial density of neutral gas. Considering the movement of all ionized electrons, the generated transverse electron current can be expressed as

$$
J(t)=e \int_{-\infty}^{t} v\left(t, t^{\prime}\right) \exp \left[-v\left(t-t^{\prime}\right)\right] d N_{e}\left(t^{\prime}\right),
$$

where $e$ is the electron charge, $d N_{e}\left(t^{\prime}\right)$ represents the change of the electron density in the interval between $t^{\prime}$ and $t^{\prime}+d t^{\prime}, v\left(t, t^{\prime}\right)=-\frac{e}{m} \int_{t}^{t} \mathrm{~d} t^{\prime \prime} E\left(t^{\prime \prime}\right)$ can be seen as the velocity of an electron at time instant $t$ which is born at the ionization instant $t^{\prime}$, and $v$ is the phenomenological collision rate $\left(v \approx 5 \mathrm{ps}^{-1}\right)$. The time-dependent electron current $J(t)$ generates an electromagnetic pulse at $\mathrm{THz}$ frequencies. The electric field of the generated $\mathrm{THz}$ pulse is proportional to the derivative of the electron current $J(t)$ :

$$
E_{\mathrm{THz}}(t) \propto \frac{d}{d t}[J(t)] .
$$

Finally, the $\mathrm{THz}$ radiation spectrum is obtained by the Fourier transform of $E_{\mathrm{THz}}(t)$, i.e., $E_{\mathrm{THz}}(\omega)=\operatorname{FFT}\left[E_{\mathrm{THz}}(t)\right]$. The THz yield is calculated as $U_{\mathrm{THz}} \propto \int_{0}^{\omega_{t h}} E_{\mathrm{THz}}^{2}(\omega) \mathrm{d} \omega$, where $\omega_{\mathrm{h}}$ $=100 \mathrm{THz}$ is the upper limit of the $\mathrm{THz}$ spectrum considered in our simulation.

In Fig. 5(c), we present the calculated THz yield as a function of the relative phase $\Delta \varphi$. As can be seen, the dependence agrees quantitatively with the experimental results shown in Fig. 5(b). The intensity variation period is $2 \pi$, instead of the period of $\pi$ observed in the two-color pumping situation $[13,20]$. This is due to the fact that a change of $\Delta \varphi$ by $\pi$ leads to a mirror symmetrical synthesized laser field in case of two-color excitation. As a result, this leads to a polarity inversed $\mathrm{THz}$ waveform of identical amplitude, which corresponds to the same $\mathrm{THz}$ energy $[13,32]$. In the situation of three-color sawtooth-like wave pumping, when $\Delta \varphi$ is changed by $\pi$ the THz amplitude becomes different since the $266 \mathrm{~nm}$ field breaks the mirror 
symmetry of the $800 \mathrm{~nm}$ and $400 \mathrm{~nm}$ field. Consequently, the variation period in Fig. 5(c) becomes to $2 \pi$. In Fig. 7(b), the simulated $\mathrm{THz}$ amplitude is compared with the experimental results, where a good agreement is also obtained. Based on a similar analysis the period with respect to $\Delta \gamma$ is also $2 \pi$.

\section{Conclusion}

In conclusion, we demonstrated a simple and robust inline optical setup enabling precise control of the relative phases between three harmonic femtosecond laser fields, providing a sawtoothlike wave. It is based on the widely available femtosecond laser pulses at $800 \mathrm{~nm}$. It was observed that the $\mathrm{THz}$ radiation amplitude produced in air with this phase-optimized three-color sawtooth-like wave is enhanced by $80 \%$ compared to that produced with two-color laser fields. The coherent control of azimuth angle, the polarity and amplitude of the enhanced $\mathrm{THz}$ pulse through a variation of the relative phases $\Delta \varphi$ or $\Delta \gamma$ of the three-color fields is in good agreement with the transient electron current model. Well controlled production of intense THz pulses of ultrashort duration (close to a single cycle pulse) and concomitant broadband spectrum should be beneficial for ultrafast dynamics application in the THz domain. Moreover, this simple and robust 3-color optical field synthesizer with attosecond precision can find wide applications in the domain of femtosecond laser-material interaction, such as high-order harmonic generation $[34,35]$, generation of white-light continuum in the mid-IR and UV regime [36-38], and fieldfree molecule alignment and orientation [39], where three-color femtosecond pulse excitation has been found to be much more efficient and beneficial.

\section{Acknowledgement}

We acknowledge the support of National Natural Science Foundation of China (Grants No. 12034013, 11604205, 11904232), Innovation Program of Shanghai Municipal Education Commission (Grant No. 2017-01-07-00-07-E00007), and Shanghai Municipal Science and Technology Commission (No. 17060502500). C. Lu acknowledges the support by Talent Program of Shanghai University of Engineering Science. 


\section{References:}

[1] H. Hamster, A. Sullivan, S. Gordon, W. White, and R.W. Falcone, "Subpicosecond, electromagnetic pulses from intense laser-plasma interaction,” Phys. Rev. Lett. 71(17), 2725-2728 (1993).

[2] D. J. Cook and, R. M. Hochstrasser, "Intense terahertz pulses by four-wave rectification in air," Opt. Lett. 25(16), 1210-1212 (2000).

[3] K. Y. Kim, A. J. Taylor, J. H. Glownia, and G. Rodriguez, "Coherent control of terahertz supercontinuum generation in ultrafast laser-gas interactions," Nat. Photonics 2, 605-609 (2008).

[4] E. Matsubara, M. Nagai, and M. Ashida, "Ultrabroadband coherent electric field from far infrared to 200 THz using air plasma induced by 10 fs pulses,” Appl. Phys. Lett. 101(1), 011105 (2012).

[5] A. Nahata, A. S. Weling, and T. F. Heinz, "A wideband coherent terahertz spectroscopy system using optical rectification and electrooptic sampling,” Appl. Phys. Lett. 69(16), 2321-2323 (1996).

[6] Q. Wu and X.-C. Zhang, "Freespace electrooptic sampling of terahertz beams," Appl. Phys. Lett. 67(24), 3523-3525 (1995).

[7] T. Löffler, M. Kreß, M. Thomson, T. Hahn, N. Hasegawa and H. G Roskos, "Comparative performance of terahertz emitters in amplifier-laser-based systems," Semicond. Sci. Technol. 20, S134S141 (2005).

[8] C. D’Amico, A. Houard, M. Franco, B. Prade, and A. Mysyrowicz, A. Couairon, and V. T. Tikhonchuk, "Conical Forward THz Emission from Femtosecond-Laser-Beam Filamentation in Air," Phys. Rev. Lett. 98(23), 235002 (2007).

[9] T. I. Oh, Y. S. You, N. Jhajj, E. W. Rosenthal, H. M. Milchberg, and K. Y. Kim, "Intense terahertz generation in two-color laser filamentation: energy scaling with terawatt laser systems,” New J. Phys. 15, 075002 (2013).

[10] I. Babushkin, W. Kuehn, C. Köhler, S. Skupin, L. Bergé, K. Reimann, M. Woerner, J. Herrmann, and T. Elsaesser, "Ultrafast spatiotemporal dynamics of Terahertz generation by ionizing two-color femtosecond pulses in gases," Phys. Rev. Lett. 105(5), 053903 (2010).

[11] Y. J. Yoo, D. Jang, and K. Y. Kim, "Highly enhanced terahertz conversion by two color laser filamentation at low gas pressures,” Opt. Express 27(16), 22663-22673 (2019).

[12] Y. J. Yoo, D. Kuk, Z. Zhong, and K. Y. Kim, "Generation and characterization of strong Terahertz fields from kHz laser filamentation,” IEEE Selected Topic in Quantum Electronics, 23, 8501007 (2017). [13] X. Xie, J. Dai, and X.-C. Zhang, “Coherent control of THz wave generation in ambient air,” Phys. Rev. Lett. 96(7), 075005 (2006).

[14] H. Wen and A. M. Lindenberg, "Coherent Terahertz polarization control through manipulation of electron trajectories,” Phys. Rev. Lett. 103(2), 023902 (2009).

[15] J. Dai, N. Karpowicz, and X. C Zhang, “Coherent polarization control of Terahertz waves generated from two-color laser-induced gas plasma," Phys. Rev. Lett. 103(2), 023001 (2009).

[16] Z. Zhang, Y. Chen, S. Cui, F. He, M. Chen, Z. Zhang, J. Yu, L. Chen, Z. Sheng, and J. Zhang, "Manipulation of polarizations for broadband terahertz waves emitted from laser plasma filaments," Nat. Photonics 12, 554-559 (2018).

[17] F. Théberge, M. Châteauneuf, G. Roy, P. Mathieu, and J. Dubois, "Generation of tunable and broadband far-infrared laser pulses during two-color fifilamentation,” Phys. Rev. A 81(3), 033821 (2010). 
[18] I. Babushkin, S. Skupin, A. Husakou, C. Köhler, E. Cabrera-Granado, L. Bergé, and J. Herrmann, "Tailoring terahertz radiation by controlling tunnel photoionization events in gases," New J. Phys. 13, 123029 (2011).

[19] N. V. Vvedenskii, A. I. Korytin, V. A. Kostin, A. A. Murzanev, A. A. Silaev, and A. N. Stepanov, "Two-color laser-plasma generation of Terahertz radiation using a frequency-tunable half harmonic of a femtosecond pulse," Phys. Rev. Lett. $112(5), 055004$ (2014).

[20] K.-Y. Kim, J. H. Glownia, A. J. Taylor, and G. Rodriguez, "Terahertz emission from ultrafast ionizing air in symmetry-broken laser fields," Opt. Express 15(8), 4577-4584 (2007).

[21] V. A. Andreeva, O. G. Kosareva, N. A. Panov, D. E. Shipilo, P. M. Solyankin, M. N. Esaulkov, P. González de Alaiza Martínez, A. P. Shkurinov, V. A. Makarov, L. Bergé, and S. L. Chin, "Ultrabroad Terahertz spectrum generation from an air-based filament plasma,” Phys. Rev. Lett. 116(6), 063902 (2016).

[22] L. Bergé, S. Skupin, C. Köhler, I. Babuchkin, and J. Herrmann, “3D numerical simulations of THz generation by two-color laser filaments,” Phys. Rev. Lett. 110(7), 073901 (2013).

[23] V. A. Kostin, I. D. Laryushin, A. A. Silaev, and N. V. Vvedenskii, "Ionization-induced multiwave mixing: Terahertz generation with two-color laser pulses of various frequency ratios," Phys. Rev. Lett. 117 (3), 035003 (2016).

[24] L. L. Zhang, W. M. Wang, T. Wu, R. Zhang, S. J. Zhang, C. L. Zhang, Y. Zhang, Z. M. Sheng, and X. C. Zhang, "Observation of Terahertz radiation via the two-color laser scheme with uncommon frequency ratios," Phys. Rev. Lett. 119(23), 235001 (2017).

[25] P. González de Alaiza Martínez, I. Babushkin, L. Bergé, S. Skupin, E. Cabrera-Granado, C. Köhler, U. Morgner, A. Husakou, and J. Herrmann, "Boosting Terahertz generation in laser-field ionized gases using a sawtooth wave shape,” Phys. Rev. Lett. 114(18), 183901 (2015).

[26] C. Lu, C. Zhang, L. Zhang, X. Wang, and S. Zhang, "Modulation of terahertz-spectrum generation from an air plasma by tunable three-color laser pulses," Phys. Rev. A 96(5), 053402 (2017).

[27] V. A. Kostin, I. D. Laryushin, N. V. Vvedenskii, “Generation of Terahertz radiation by multicolor ionizing pulses,” JETP Lett. 112(2) 77-83 (2020).

[28] A. Wirth, M. Th. Hassan, I. Grguraš, J. Gagnon, A. Moulet, T. T. Luu, S. Pabst, R. Santra, Z. A. Alahmed, A. M. Azzeer, V. S. Yakovlev, V. Pervak, F. Krausz, E. Goulielmakis, "Synthesized light transients," Science 334(6053), 195-200 (2001).

[29] C. Burger, W. F. Frisch, T. M. Kardaś, M. Trubetskov, V. Pervak, R. Moshammer, B. Bergues, M. F. Kling, and P. Wnuk, "Compact and flexible harmonic generator and three-color synthesizer for femtosecond coherent control and time-resolved studies," Opt. Express 25(25), 31130-31139 (2017).

[30] V. Vaičaitis, O. Balachninaite, U. Morgner, and I. Babushkin, “Terahertz radiation generation by three-color laser pulses in air filament,” J. Appl. Phys. 125, 173103 (2019).

[31] J. D. Bagley, C. D. Moss, S. A. Sorenson, J. A. Johnson, "Laser-induced plasma generation of terahertz radiation using three incommensurate wavelengths,” J. Phys. B, 51, 144004 (2018).

[32] J. Dai and X. C. Zhang, “Terahertz wave generation from gas plasma using a phase compensator with attosecond phase-control accuracy,” Appl. Phys. Lett. 94(2), 021117 (2009). 
[33] P. B. Corkum, N. H. Burnett, and F. Brunel, "Above threshold ionization in the long wavelength limit," Phys. Rev. Lett. 62(11), 1259 (1989).

[34] P. Wei, J. Miao, Z. Zeng, C. Li, X. Ge, R. Li, and Z. Xu, "Selective enhancement of a single harmonic emission in a driving laser field with subcycle waveform control," Phys. Rev. Lett. 110(23), 233903 (2013).

[35] C. Jin, G. Wang, H. Wei, A.-T. Le, and C. D. Lin, "Waveforms for optimal sub-keV high-order harmonics with synthesized two- or three-colour laser fields," Nat. Commun. 5, 4003 (2014).

[36] P. B. Petersen and A. Tokmakoff, "Source for ultrafast continuum infrared and terahertz radiation," Opt. Lett. 35(12), 1962-1964 (2010).

[37] Y. Kida, K. Sakamoto, and T. Imasaka, "High-energy multicolor femtosecond pulses in the deep-ultraviolet generated through four-wave mixing induced by three-color pulses,” Appl. Phys. B 122, 214 (2016).

[38] V. A. Kostin and N. V. Vvedenskii, "Generation of few- and subcycle radiation in midinfrared-todeep-ultraviolet range during plasma production by multicolor femtosecond pulses,” Phys. Rev. Lett. 120(6), 065002 (2018).

[39] S. A. Zhang, J. H. Shi, H. Zhang, T.Q. Jia, Z. G. Wang, and Z. R. Sun, "Field-free molecular orientation by a multicolor laser field," Phys. Rev. A 83(2), 023416 (2011). 


\section{Figure 1}

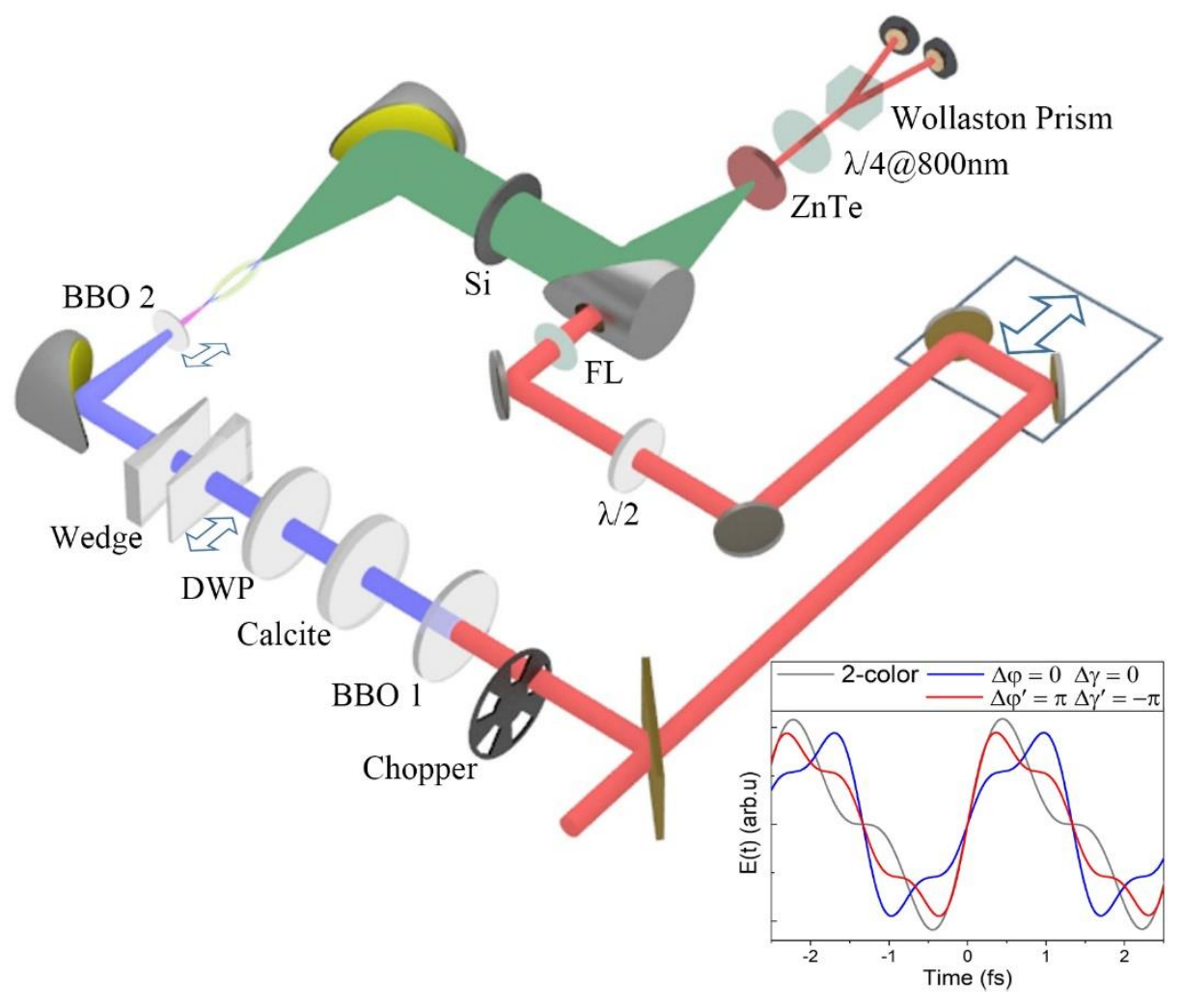

Fig. 1. Schematic experimental setup. The femtosecond laser pulses were split into pump and probe beams with a beam splitter. The BBO 1 and BBO 2 were used for second harmonic and third harmonic generation. The calcite plate cut at $90^{\circ}$ imposes a negative group delay for the $800 \mathrm{~nm}$ and $400 \mathrm{~nm}$ pulse, in order to compensate the positive delay induced by other optical elements. The dual wavelength plate (DWP) rotates the polarization plane of the 400 $\mathrm{nm}$ by $90^{\circ}$ while keeping the polarization of the $800 \mathrm{~nm}$ unchanged. The insertion of the fused silica wedge was controlled by a mechanic motor. The THz radiation emitted by the air plasma was detected by the electrical-optical sampling system composed of the ZnTe crystal, the quarter wave-plate, the Wollaston prism, and the balanced detector. Inset: the calculated synthesized laser field by two colors (gray line) and the sawtooth-like wave composed of three colors (red and blue lines). 
Figure 2

(a)

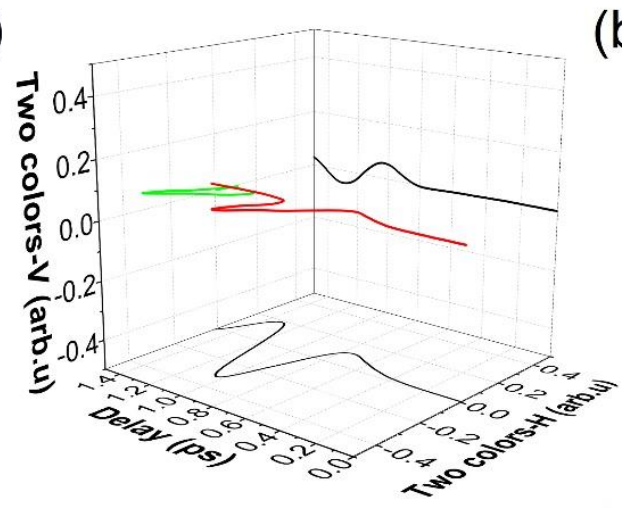

(c)

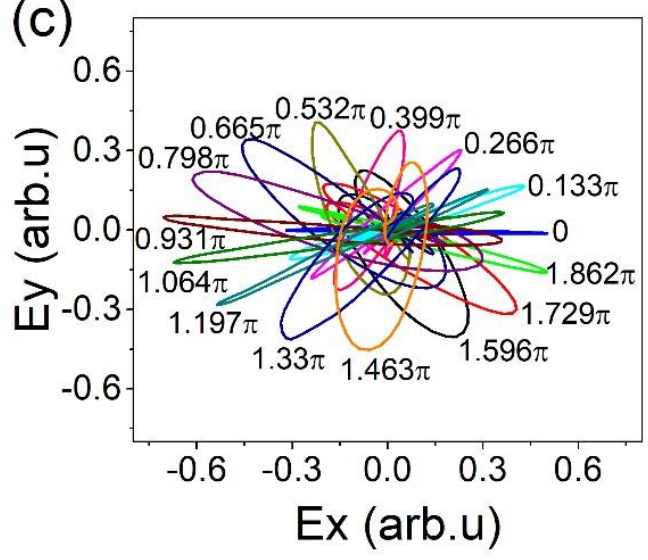

(b)

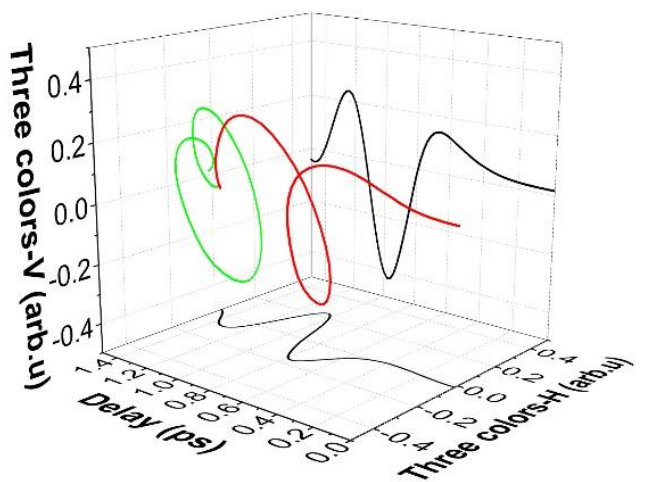

(d)

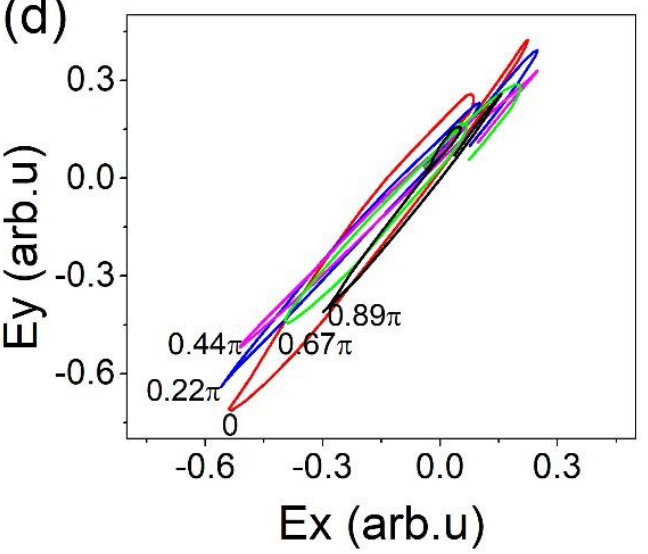

Fig. 2. (a) and (b), Temporal waveform of the terahertz field produced by the two-color wave and sawtooth-like threecolor wave. Both horizontal and vertical components of the THz electric field are presented. (c) and (d), Trajectories of the THz electric field in the $\mathrm{x}-\mathrm{y}$ plane as a function of the phases $\Delta \varphi$ and $\Delta \gamma$. 


\section{Figure 3}

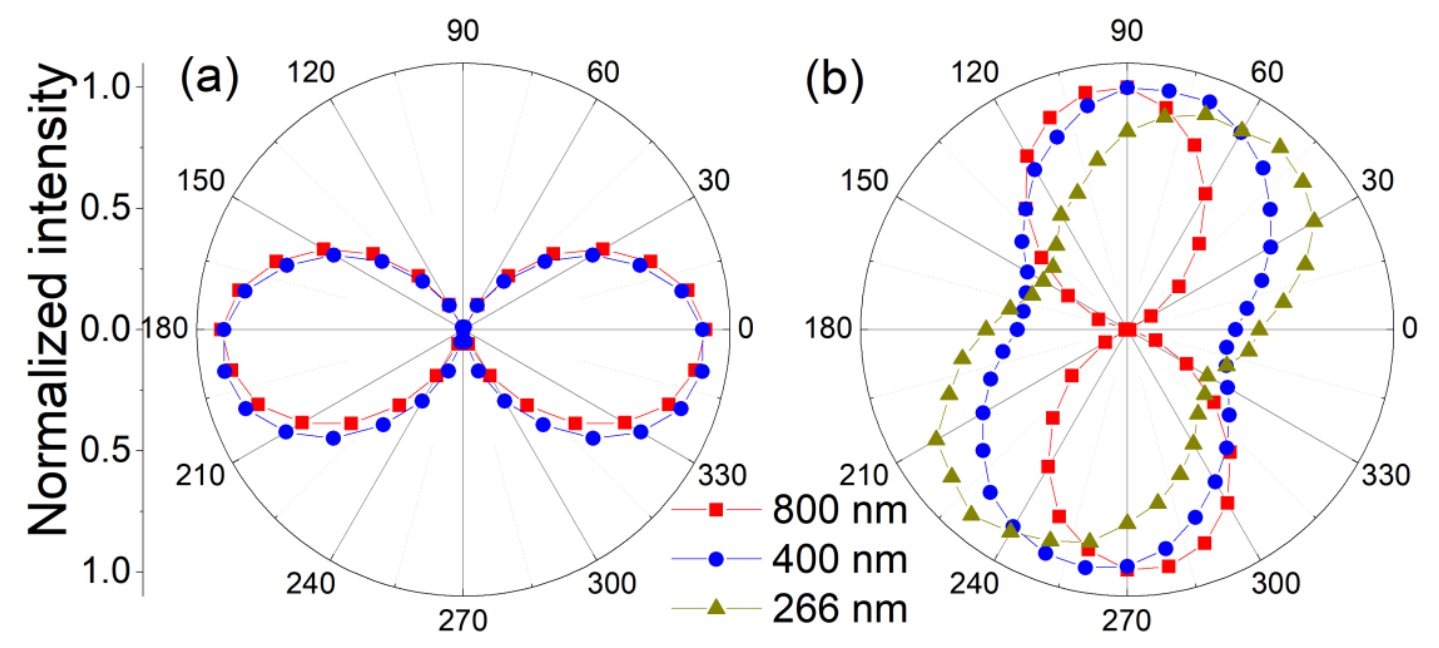

Fig. 3. Measurement of the polarization of the optical fields before (a) and after the SFG BBO crystal (b). The energies of the 800,400 , and $266 \mathrm{~nm}$ pulses are measured as a function of the rotation angle of a Glan-Taylor polarizer. 


\section{Figure 4}

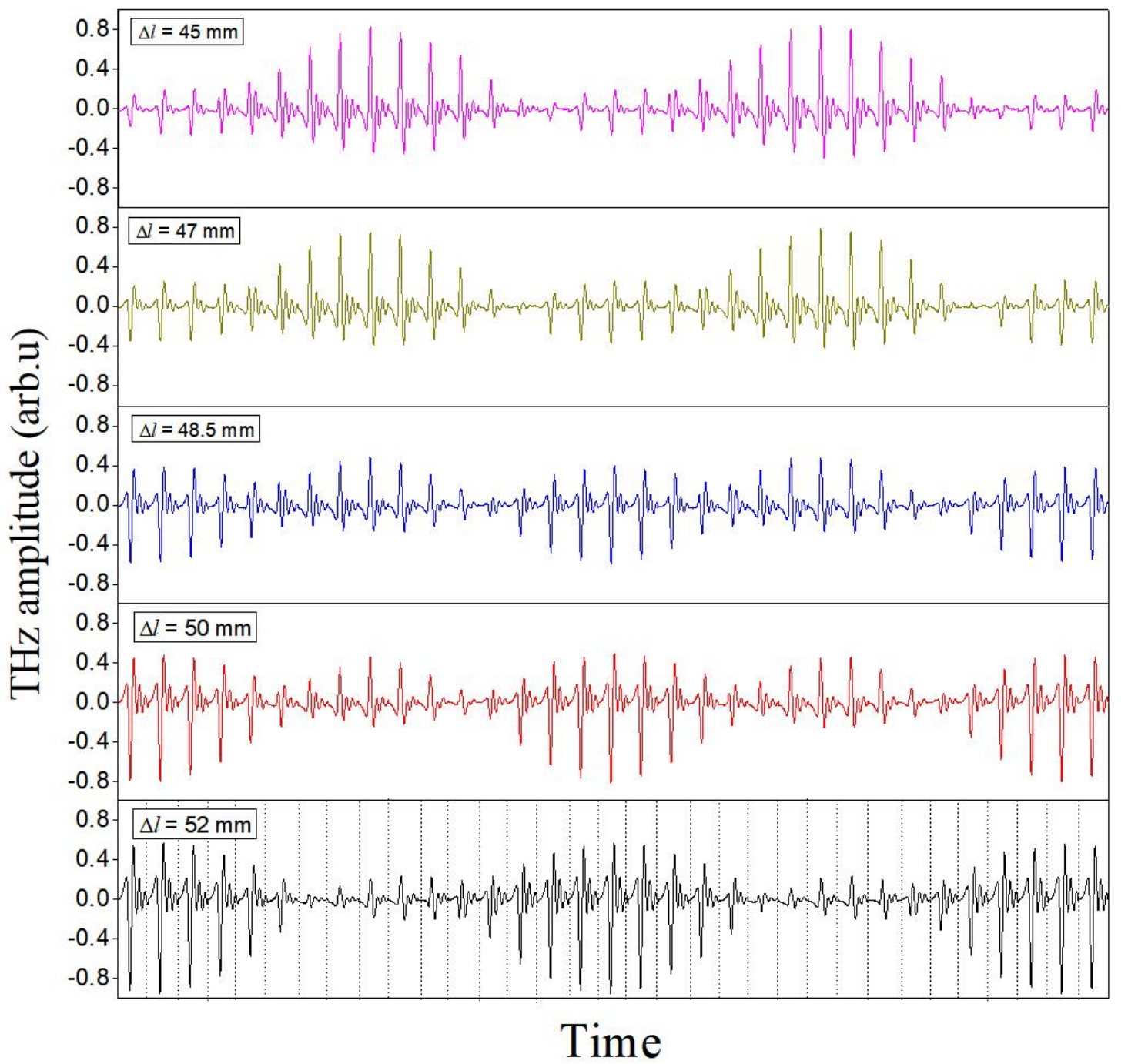

Fig. 4. Temporal waveform of the terahertz pulse as a function of the phase $\Delta \varphi$ for 5 different BBO-2-focus distances $(\Delta \gamma)$. Form (a) to (e), the BBO-2-focus distance was increased by an increment of $1.5 \mathrm{~mm}$ or $2 \mathrm{~mm}$, corresponding to a $\Delta \gamma$ increment of $0.167 \pi$ or $0.222 \pi$. In each panel, 33 independent $\mathrm{THz}$ waveforms are presented for increasing $\Delta \varphi$ with an increment of $0.133 \pi(0.03 \mathrm{~mm}$ of fused silica thickness). For each THz waveform measurement, a temporal window of $3 \mathrm{ps}$ has been presented. The square of the amplitude of the above $\mathrm{THz}$ waveforms are presented in Fig. 5 (b) in the manuscript. 
Figure 5
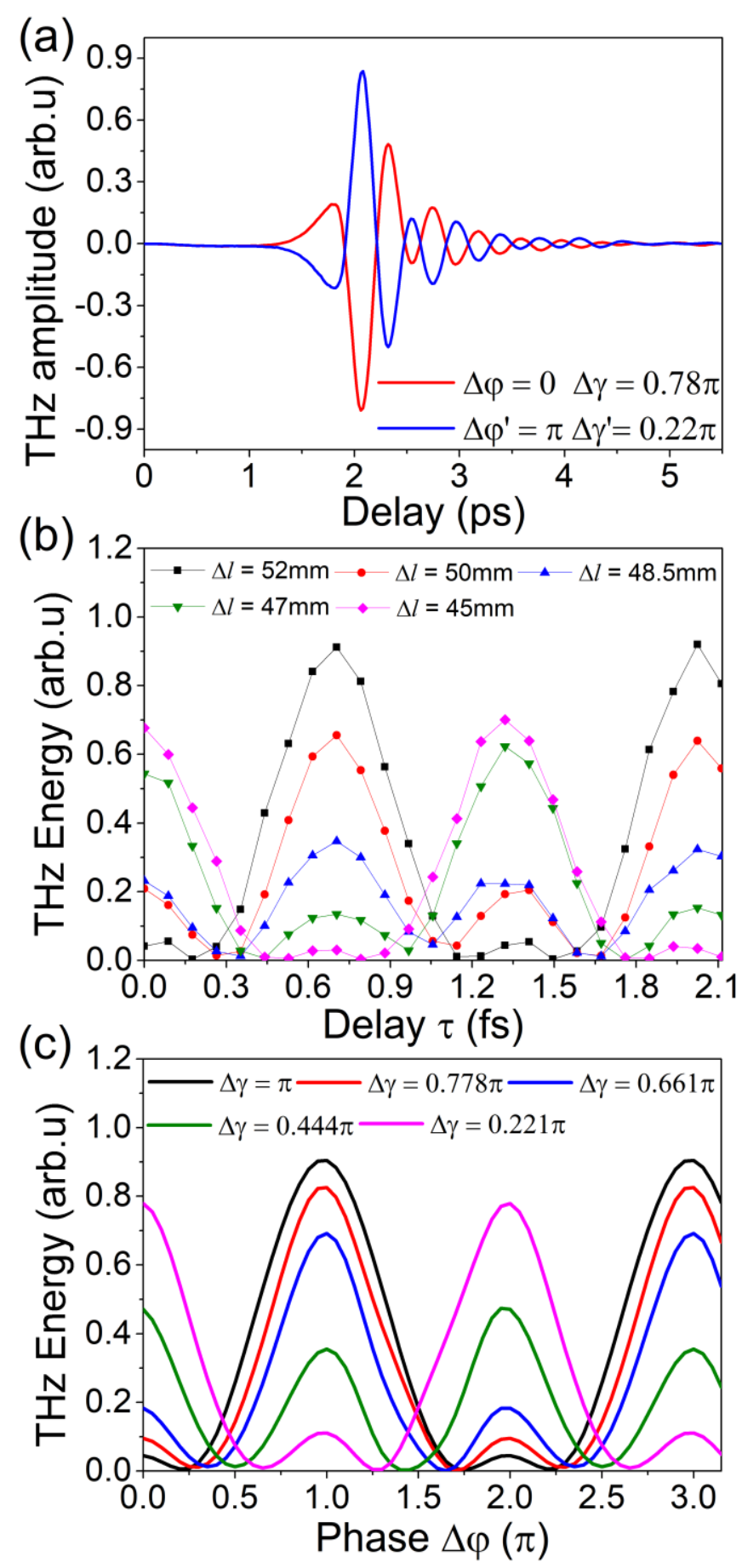

Fig. 5. (a) Experimental Terahertz temporal waveforms with opposite polarities obtained with different combination of $\Delta \varphi$ and $\Delta \gamma$. (b) Energy of the THz pulse as a function of the relative delay between the $800 \mathrm{~nm}$ and $400 \mathrm{~nm}$ laser field, while BBO-2-focus distance (corresponding to $\Delta \gamma$ ) was fixed at 5 different values. (c), THz energy from numerical simulations vs. relative phase $\Delta \varphi$. 


\section{Figure 6}

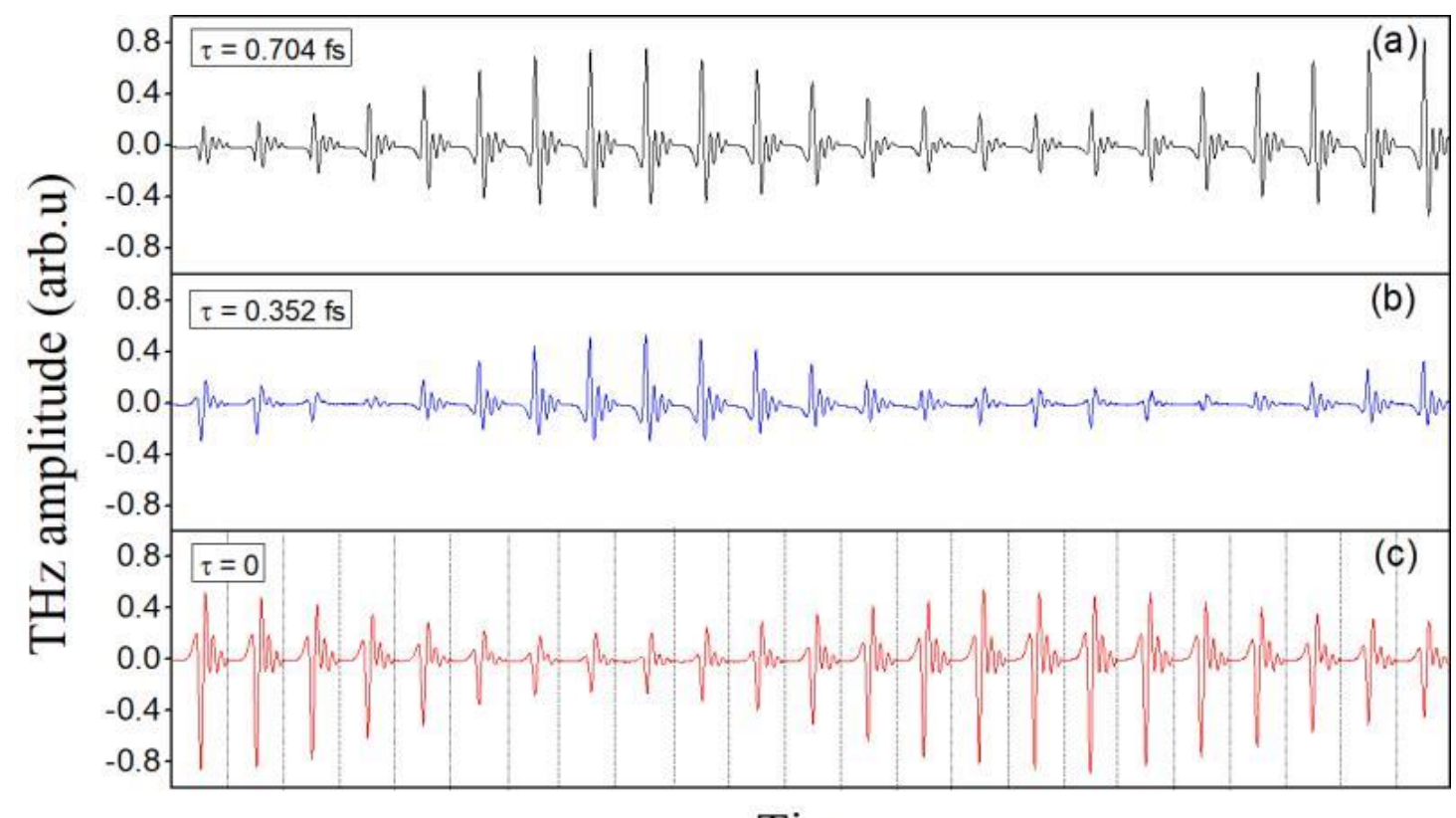

Time

Fig. 6. Temporal waveform of the terahertz pulse as a function of the phase $\Delta \gamma$ for 3 different thicknesses of the fused silica wedge $(\Delta \varphi)$. From (a) to (c), the thickness of the fused silica wedge was increased by an increment of $0.12 \mathrm{~mm}$, corresponding to an increment of the time delay between the $800-\mathrm{nm}$ and $400-\mathrm{nm}$ pulse by $0.352 \mathrm{fs}$. In each panel, 23 independent $\mathrm{THz}$ waveforms are presented for increasing $\Delta \gamma$ with an increment of $0.111 \pi$, by displacing the position of the BBO 2 with respect to the focus by $\Delta l$ of $1 \mathrm{~mm}$. For each $\mathrm{THz}$ waveform measurements, a temporal window of 3 ps has been presented. The amplitude of the above THz waveforms is presented in Fig. 7(a) in the manuscript. 
Figure 7

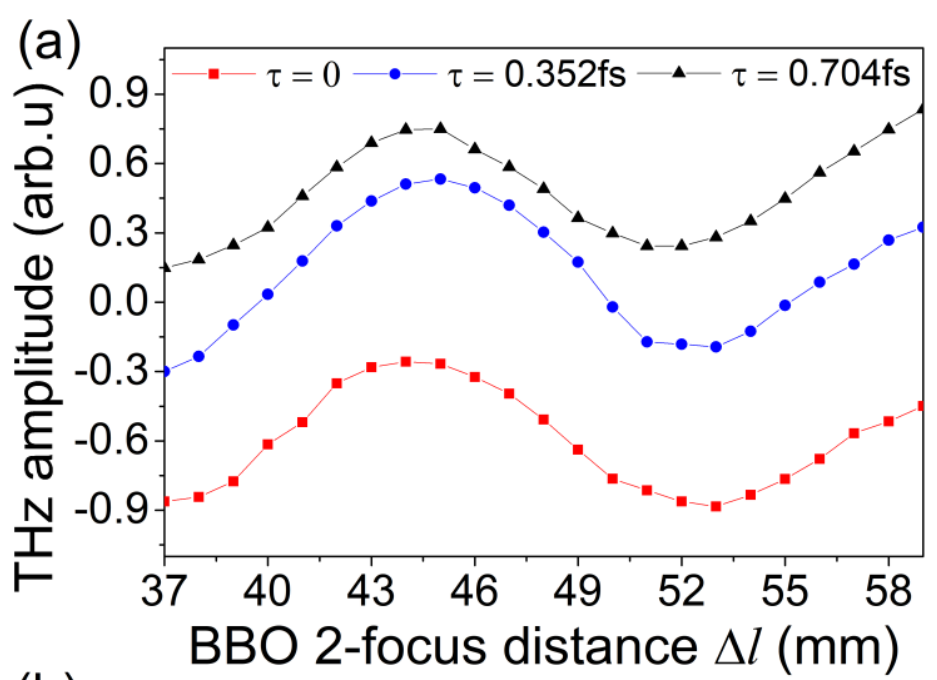

(b)

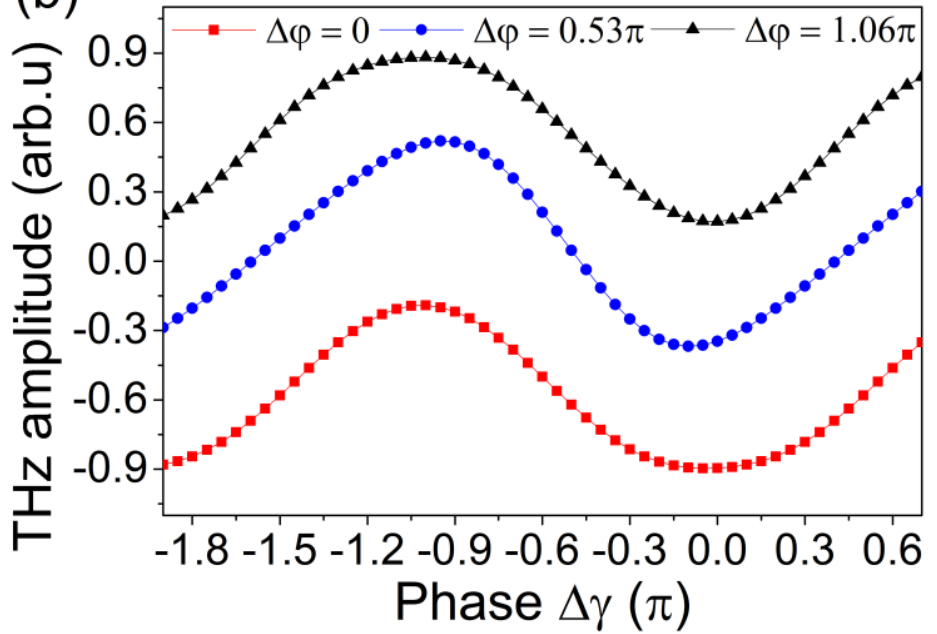

Fig. 7. (a) Amplitude of the THz pulse as a function of the BBO-2-focus distance $\Delta l$ defining $\Delta \gamma$, with delay $\tau$ (corresponding to phase $\Delta \varphi$ ) fixed at 3 different values. (b) THz amplitude from numerical simulations vs. relative phase $\Delta \gamma$. 


\section{Appendix: Phase relationship between the three-color laser fields}

In this appendix, we introduce the expression (1) used in the manuscript and describe the relationship between the phase terms and the experimental parameters. First, we introduce the expression for the three-color laser field at the focus with our optical layout. Considering the optical setup in Fig. 1 of the manuscript, the laser field can be written as:

$E(t)=\exp \left(\frac{-2 \ln 2 t^{2}}{\tau^{2}}\right) \times\left[E_{800} \sin \left(\omega_{0} t\right)+E_{400} \sin \left(2 \omega_{0} t+\varphi+\alpha\right)+E_{266} \sin \left(3 \omega_{0} t+\varphi+\beta+\gamma\right)\right]$.

Here, $t$ is the time variable, $\tau$ is the intensity full width at half-maximum, $\omega_{0}=2 \pi c / \lambda_{800}$ is the fundamental frequency. $E_{800}, E_{400}$, and $E_{266}$ are the amplitude of the fundamental, second harmonic and third harmonics. Now we explain the phase terms.

(i) The phase $\varphi$ denotes the phase difference between the $400 \mathrm{~nm}$ and $800 \mathrm{~nm}$ field introduced by the movable fused silica wedge. It is related to the thickness $d$ of the wedge by $\varphi=2 \pi\left(n_{400}-n_{800}\right) d / \lambda_{400}$, where $n_{400}$ and $n_{800}$ are the refractive indices of fused silica for 400-nm and 800-nm light pulses.

(ii) The phase $\alpha$ presents the phase difference introduced by all other transmission elements from the BBO 1 to the focus. Five elements contribute to this phase difference: (1) the second harmonic generation crystal (BBO 1), (2) the calcite plate, (3) the dualwavelength plate, (4) the sum frequency generation crystal (BBO 2), (5) the optical pass in air between the BBO 1 and the focus. For a given BBO-1-focus distance (the case of all our experiments), the above 5 contributions of the phase difference are constant. Therefore, it can be represented by a phase term $\alpha$.

(iii) For the sum-frequency generation inside the second BBO crystal, the phase difference $\varphi$ will be transferred to the $266 \mathrm{~nm}$ pulse accompanying the SFG generation. Therefore, in the phase of the $266 \mathrm{~nm}$ field there also exists the term $\varphi$.

(iv) There are two other elements contributing to the relative phase of the $266 \mathrm{~nm}$ laser field. The first element is the SFG crystal (BBO 2), which introduces a phase constant $\beta$ determined by the BBO thickness. The second contribution is the optical pass in air between the rear surface of BBO 2 and the focus. The second contribution can be expressed as $\gamma=2 \pi\left(n_{400}-n_{266}\right) l / \lambda_{266}$, where $l$ is the BBO 2-focus distance, $n_{400}$ and $n_{266}$ are the refractive indices of air for 400-nm and 266-nm pulses. Therefore, one can change the position of the BBO 2 to control $\gamma$. A change of $l$ by $10 \mu \mathrm{m}$ corresponds to a phase change of $\Delta \gamma=0.0011 \pi$.

In view that $\alpha$ and $\beta$ are both constant in our experiments and that $\varphi$ and $\gamma$ are variables, in order to write the expression of electric field to be compitable with that of the sawtooth wave in Ref. [25], we can always choose special $\varphi_{0}$ and $\gamma_{0}$ to fufill the following relatioship :

$$
\varphi_{0}+\alpha=\pi,
$$




$$
\varphi_{0}+\gamma_{0}+\beta=0
$$

In this case, the phase change induced by tranlation of the optical wedge or displacement of the BBO 2 can be expressed as :

$$
\begin{aligned}
\Delta \varphi & =\varphi-\varphi_{0}, \\
\Delta \gamma & =\gamma-\gamma_{0} .
\end{aligned}
$$

As a result, the electric field now reads as

$E(t)=\exp \left(\frac{-2 \ln 2 t^{2}}{\tau^{2}}\right) \times\left[E_{800} \sin \left(\omega_{0} t\right)+E_{400} \sin \left(2 \omega_{0} t+\pi+\Delta \varphi\right)+E_{266} \sin \left(3 \omega_{0} t+\Delta \varphi+\Delta \gamma\right)\right]$ 